\title{
Long QT syndrome and sudden unexpected infant death
}

\author{
Chantal Van Niekerk, ${ }_{1}^{1,2}$ Barbara Ströh Van Deventer, ${ }^{3}$ Lorraine du Toit-Prinsloo ${ }^{3}$
}

${ }^{1}$ Department of Chemical Pathology, National Health Laboratory Service, Johannesburg, Gauteng, South Africa

${ }^{2}$ Department of Chemical Pathology, University of Pretoria, Pretoria, South Africa ${ }^{3}$ Department of Forensic Medicine, University of Pretoria, Pretoria, South Africa

\section{Correspondence to}

Dr Chantal Van Niekerk, R3-43 Pathology Building, Prinshof Campus, University of Pretoria, Pretoria 0002, South Africa; chantal.vanniekerk@up.ac.za

Received 16 December 2016 Revised 1 June 2017 Accepted 5 June 2017 Published Online First 15 June 2017

\section{CrossMark}

To cite: Van Niekerk $C$ Van Deventer BS, du ToitPrinsloo L. J Clin Pathol 2017:70:808-813.

\section{ABSTRACT}

Long QT syndrome (LQTS) is an inheritable primary electric disease of the heart characterised by abnormally long QT intervals and a propensity to develop atrial and ventricular tachyarrhythmias. It is caused by an inherited channelopathy responsible for sudden cardiac death in individuals with structurally normal hearts. Long QT syndrome can present early in life, and some studies suggest that it may be associated with up to $20 \%$ of sudden unexplained infant death (SUID), particularly when associated with external stressors such as asphyxia, which is commonly seen in many infant death scenes. With an understanding of the genetic defects, it has now been possible to retrospectively analyse samples from infants who have presented to forensic pathology services with a history of unexplained sudden death, which may, in turn, enable the implementation of preventative treatment for siblings previously not known to have pathogenic genetic variations. In this viewpoint article, we will discuss SUID, LQTS and postmortem genetic analysis.

\section{SUDDEN UNEXPECTED INFANT DEATH}

In most countries, sudden and unexpected death cases will be referred for routine medicolegal autopsy. Unfortunately, $70 \%$ to $80 \%$ of sudden unexpected deaths in infants (SUDIs) will remain unexplained, even after thorough investigation, which include a detailed postmortem examination including macroscopic examination with evisceration of all organs and all ancillary investigations such as histology, microbiology, virology and toxicology. ${ }^{1-3}$ The Centre for Disease Control and Prevention estimated in 2016 that 3500 infants die suddenly and unexpectedly each year in the USA. ${ }^{4}$ A review study conducted in Wales reported the approximate prevalence of SUDI was $14 \%$ of all infant deaths recorded over a 2-year period (20102012). ${ }^{5}$ These unexplained deaths were previously defined as sudden infant death syndrome (SIDS). ${ }^{6-9}$

In 2013, Byard indicated a possible diagnostic shift in SIDS cases. During the 1990s, the continued monitoring of diagnostic practices and trends in infant deaths revealed the extent to which pathologists contributed to this diagnostic shift. ${ }^{10,11}$ An increased awareness of the infant's position in relation with many of these sudden deaths enabled the pathologists to identify more cases of accidental asphyxia in relation to unsafe sleeping environments. Furthermore, Byard also documented an opposing component of the diagnostic shift, which involved the subjective reassignment of causes of death. ${ }^{12}$ A specific trend was detected where many pathologists refrained from attributing the cause of death to SIDS and rather used terms such as undetermined cause of death or asphyxia-related death. ${ }^{12}$ Reasons for this shift include the absence of pathognomonic diagnostic features for SIDS and the insufficient findings that may be present in cases of accidental or intentional smothering. ${ }^{12-15}$ Pathologists have rather taken to determining these deaths as sudden unexplained infant deaths (SUIDs), which are defined as 'the death of an infant less than one year of age in which investigation, autopsy, medical history review and appropriate laboratory testing fail to identify a specific cause of death. SUID includes cases that meet the definition of sudden infant death syndrome. ${ }^{16}$

\section{AETIOLOGY OF SUID}

Studies show SUID occurred more frequently in infants between the age of 2 and 4 months and rarely after the age of 8 months. ${ }^{1,3,17-20}$ Death apparently occurs during periods of sleep, suddenly and without warning. ${ }^{1,17}$ A uniformly accepted triple-risk model was first introduced in 1994 by Filliano and Kinney, and highlighted the interaction of multiple risk factors that increase the probability of SUID. ${ }^{21}$ These risk factors are divided into three groups: a vulnerable infant, a critical developmental stage and exogenous stressors. ${ }^{21}$ Current theories still suggest that SUID is a complex event and infants may die when risk factors in each of these groups occur at the same time: a vulnerable infant (which can include an underlying genetic mutation/predisposition) in a critical developmental stage (peaks at 3 months) with an exogenous stressor such as asphyxia challenges from unsafe sleeping practices, soft bedding, the exposure to second-hand smoke as well as bacterial and viral infections. ${ }^{3,17-22}$

In the 1990s, there was a decrease in the number of SUID cases, which could probably be attributed to the introduction of the 'back-to-sleep' campaign. However, since then, the SUID rate has remained stable and is the number one cause of death in postneonatal infants in most developed countries. ${ }^{3,18,19}$ The large number of published studies strongly suggests that SUID may be multifactorial and may include metabolic and genetic disorders, as well as deficits in serotonin receptors in the brainstem, ${ }^{23,24}$ which motivates for the continuous research into possibly preventable causes. ${ }^{1,3,17-19}$ Fortunately, with the rapid development in technology and continued studies on genetic risk factors, postmortem molecular analysis proved to be an invaluable tool in determining a possible cause of death in many SUID cases. ${ }^{25,26}$ 
A postmortem genetic study conducted by Wang et al showed that in their cohort of infants, African-Americans had the highest risk of dying suddenly, followed by Hispanics and Caucasians, with the Asian population at smallest risk. ${ }^{25}$ Arnestad et al suggested an intriguing hypothesis with regard to possible modulating factors involving specific genetic variants and the associated ethnicity of the individual. ${ }^{18}$ Comparing the ethnic/racial differences as described above with the occurrence of SUIDs indicates that the rate of SUIDs among lower income/socioeconomic deprived racial and ethnic groups showed an increase compared with groups within a higher income bracket. ${ }^{11}$ American Indians, African-Americans, Maoris from New Zealand as well as Aboriginals in Australia all have a higher incidence of SUID. ${ }^{1,17}$ No definitive explanation for this increased occurrence could be found; however, a complex interaction between genetic and environmental risk factors may be the underlying basis-in keeping with the triple-risk model.

\section{SUIDS AND CHANNELOPATHIES}

Numerous studies have been done on the association of serotonin receptor deficits in SUIDs. ${ }^{2,3,8,27}$ In addition to serotonin receptor deficits, other studies, which have also received increased attention over the past few years, have shown that one of the possible preventable causes of SUIDs is that of inherited, life-threatening cardiac arrhythmic disorders, commonly referred to as cardiac channelopathies. ${ }^{26,28-30}$ These channelopathies, which include long QT syndrome (LQTS), Brugada syndrome (BrS) and catecholaminergic polymorphic ventricular tachycardia (CPVT), are a result of pathogenic variants in genes that code for cardiac ion channels. ${ }^{25,26,28,30}$ These genes play a role in the cardiac electrical conduction physiology, thus affecting the normal heart rhythm. ${ }^{8,31-33}$

The first evidence pertaining to cardiac conduction disorder in SUIDs is that of Keeton et al, ${ }^{34}$ who in 1977 reported on the diagnosis of severe conduction disorders in six cases of acute life-threatening events (ALTE) in infants. These infants received proper treatment before any fatalities occurred. ${ }^{34}$ Data obtained from six separate studies indicate that the overall prevalence of pathogenic variants in cardiac ion-channel-related genes in SUID victims may be $20 \%$. These variants seem to have a fatal outcome when coinciding with certain stressors/triggers such as fever and asphyxia, ${ }^{18,35-39}$ which is especially relevant when considering that asphyxia is commonly encountered in SUID especially in a so-called unsafe sleeping environment. The American National Society of Genetic Counselors, ${ }^{40}$ Ackerman, ${ }^{53}$ Michaud et al, ${ }^{33}$ Arnestad $e t a l^{18}$ and Davis et $a l^{29}$ all reported that an average of $15 \%$ of SUID cases occurred due to inherited cardiac arrhythmic disorders. It was suggested that the putative cause of death in one of every five SUIDs may be the result of pathogenic variants in a cardiac ion-channel-related gene. ${ }^{13,63}$

The 'peak' age of SUIDs is commonly accepted as 3 months. ${ }^{1,3,17-20}$ However, in infants identified with a channelopathy, the age range at time of death varies greatly between each study cohort, with no peak age of death noted among all the studies. Some recorded a range between 4 days and 12 months while others recorded median ages at death varying from 2 months up to 6 months. ${ }^{18,20,25}$ The exact mechanism to which this relatively broad span of age range can be attributed to is still unknown. It should be kept in mind that the broader definition of SUID includes all infants up to the age of 1 year.

Some variants in genes linked to the different channelopathies seem to be more prevalent in certain population groups while rare in others. ${ }^{18,25}$ A number of studies indicate a higher prevalence of certain genetic variants among the Maori population, ${ }^{1,17,20}$ whereas other specific variants, especially the SCN5AH558R amino acid replacement, are associated with a higher prevalence in the Caucasian population group. ${ }^{41}$ In contrast, certain common variants found in the Hispanic and Asian populations are identified as disease-causing variants in the Caucasian population. ${ }^{18}$ The SCN5A-A572D variant, which has previously been described as disease-causing, is a common variant found in the Norwegian population. ${ }^{18}$

\section{LONG QT SYNDROME}

The channelopathy that has the strongest link to SUIDs is LQTS. ${ }^{2,26}$ LQTS is an inherited arrhythmogenic disorder associated with the ionic control of the cardiac action potential. Clinical outcomes include syncope, seizures and sudden death, especially in young and apparently healthy individuals. Of note, all LQTS features, including a postmortem examination that remains unexplained, are similar to SUID. ${ }^{2,26}$

LQTS is a genetically heterogeneous condition, with the majority of cases inherited in an autosomal dominant manner. The less common recessive forms of LQTS are associated with severe cardiac phenotypes and congenital deafness. ${ }^{31,42,43}$ The characteristics of LQTS are represented by a delayed repolarisation of the ventricular cells. This is attributed to the reduction in repolarising (outward) currents, or an increase in depolarising (inward) currents, and is associated with ECG manifestations of prolonged QT intervals and T wave abnormalities. ${ }^{43-45}$ The prevalence of inherited LQTS is estimated to be 1 in 2500 live births. ${ }^{18,26,28}$ However, reports have indicated that this number might be an underestimation since the likelihood for a misdiagnosis exists in approximately two-thirds of patients with LQTS due to the heterogeneity of the disease..$^{13,25,26,28}$ In addition, an estimated 10\%-35\% of patients present with a normal QT interval when measured on a resting 12-lead ECG. This further contributes to the underestimated prevalence of inherited LQTS in the general population. ${ }^{28,42-44,46}$ The onset of symptoms usually occurs at a mean age of 12 years, with an earlier onset of symptoms typically associated with more severe outcomes. ${ }^{42-44,47}$

To date, a significant number of genetic variations have been associated with LQTS. ${ }^{3,18,48}$ According to the Human Gene Mutation Database, more than 600 long QT variations have been identified in several ion-channel-related genes. ${ }^{49}$ Three major genes are responsible for $75 \%-90 \%$ of these variants: the potassium voltage-gated channel subfamily $Q$ member 1 (KCNQ1), the potassium voltage-gated channel subfamily $\mathrm{H}$ member 2 (KCNH2) and the sodium voltage-gated channel type $\mathrm{V}$ alpha (SCN5A) gene. ${ }^{43,44,50}$ Loss-of-function variants in KCNQ1, encoding for the ion channel that mediates the slow delayed rectifying potassium current $\left(\mathrm{I}_{\mathrm{Ks}}\right)$, cause long QT type 1 (LQT1) syndrome. Most arrhythmias experienced in LQT1 patients are triggered by exercise-related stress. ${ }^{31,33,43,51}$ Lossof-function variants in $\mathrm{KCNH} 2$, encoding for the ion channel generating the rapid delayed rectifying potassium current $\left(\mathrm{I}_{\mathrm{Kr}}\right)$ during repolarisation, cause long QT type 2 (LQT2) syndrome. In LQT2 patients, the majority of events are triggered by emotional stress. ${ }^{43,44,47}$ Gain-of-function variants in SCN5A, encoding for the sodium channel that generates the depolarising $I_{\mathrm{N}}$ sodium current, cause long QT type 3 (LQT3) syndrome. ${ }^{4,43,52-54}$ The cardiac events in LQT3 patients, which are considered the most lethal among LQTS, occur during a period of sleep/rest and have been reported in SUID cases. ${ }^{20,26,44,53}$ The higher lethality rate can be best explained by the $20 \%$ increased risk of sudden death presenting as the 
first clinical manifestation in LQT3 patients versus the 4\% risk among LQT1 and LQT2 patients. ${ }^{20,43,44}$

\section{LONG QT SYNDROME AND SUID}

Of all the channelopathies, LQTS is the most prevalent disorder associated with SUIDs ${ }^{3,20,26,55,56}$ as well as sudden death in the young. ${ }^{28,33,57,58}$ Postmortem genetic testing in SUID cases demonstrated that $13.9 \%$ of cases with identified variants in the LQTS genes have pathogenic clinical significance. ${ }^{13,28}$

A large population-based study conducted on the clinical association between a prolonged QT interval in ECGs and an increased risk of SUID analysed 33034 ECGs of healthy Italian babies, which were taken on the third or fourth day of life. ${ }^{31}$ In each case, the QT interval was measured and the infants were followed for 1 year. In total, 34 infants died, of which 24 deaths were attributed to SUID (incidence of 0.7 per 1000 live births). A prolonged QT interval was recorded in 12 of the SUID cases $(50 \%)$, whereas none of the survivors, or infants who died of other causes, demonstrated a prolonged QT interval. ${ }^{31}$ As a result, Schwartz et $a l^{31}$ calculated the OR for SUID in infants with a prolonged QT interval as 41, an OR significantly higher than that of prone posture and maternal smoking. ${ }^{13}$

A more recent follow-up study on the association of LQTS with an increased risk for SUID involved a comprehensive 19-year prospective review of ECGs, which were recorded between 15 and 28 days of life in more than 44000 infants. ${ }^{59}$ Molecular screening was performed in 28 infants who presented with a marked QT interval prolongation, which showed that 14 of these infants $(50 \%)$ were carriers of potentially pathogenic LQTS-related variants. All neonates who presented with a prolonged QT interval received successful treatment with a $\beta$-blocker (propranolol). ${ }^{59}$

An association between LQTS variations and SUID victims has been recognised by two well-known case studies: ${ }^{56,60}$ one on a SUID case and the other on an infant with documented ventricular fibrillation who survived an ALTE. These two studies ultimately paved the way for other cohort studies on SUIDs. ${ }^{56,60}$ One study showed a 5.2\% prevalence of LQTS causing variations in a study cohort of 68 SUID cases. ${ }^{35}$ Another study, composed of 201 SUID cases and 187 controls, found that 9.5\% (95\% CI 5.8 to 14.4 ) of SUID cases carried functional LQTS pathogenic variations, whereas none of the controls did. ${ }^{18} \mathrm{~A}$ third study, conducted by Wang et al, ${ }^{25}$ identified variants of probable pathogenic significance in 19 of 141 SUID cases (13.5\%).

Long QT type 3 syndrome seems particularly important in SUID cases as studies demonstrated a link between SUID and a predominance of SCN5A gene variants. ${ }^{18,19,53,54,61,62}$ In three different studies, molecular screening identified pathogenic variants linked to LQTS in a number of SUID cases, where variations in the SCN5A gene comprised respectively 50\%, 68.4\% and $50 \%$ of all identified variants. ${ }^{28,52,63}$ This could be ascribed to the known genotype-phenotype correlations that suggest patients with LQT3 (SCN5A) variants may experience a higher lethality rate, mostly occurring during sleep, compared with patients who have variants in other genes involved in LQTS. ${ }^{18,20,64}$

The SCN5A gene is a member of the voltage-gated sodium channel family, with at least nine sodium channel $\alpha$-subunits in this family identified from various human tissues. ${ }^{28,32,61}$ The genomic location of SCN $5 A$ is on the short arm of chromosome 3 at position 21 (3p21). It consists of 28 exons with an approximate span of 80000 base pairs $(80 \mathrm{~kb}) .^{31,32,41,61}$ The SCN5A gene encodes for a protein (sodium (Nav1.5) ion channel poreforming $\alpha$-subunit) of 2016 amino acids with a calculated molecular weight of $227 \mathrm{kDa}$. The voltage-gated $\mathrm{Na}^{+}$channel $\alpha$-subunit contains six transmembrane-spanning segments (S1-S6) found within each of four homologous domains (DIDIV). ${ }^{28,32,52,63}$ It is restrictively expressed in the myocardium and plays a critical role in heart excitability and conduction. ${ }^{28,31,46}$ The integral membrane protein produces the fast inward $\mathrm{Na}^{+}$ current that is responsible for the depolarising phase of the cardiac action potential. ${ }^{13,28,46}$ Variations of this gene cause a persistent $\mathrm{Na}^{+}$current with a subsequent prolongation of the ventricular action potential, essentially resulting in an inherited predisposition to ventricular arrhythmias and sudden death, seen in several cardiac diseases, including LQT3. ${ }^{29,54,65-67}$

\section{POSTMORTEM GENETIC TESTING AND SUID}

Postmortem genetic testing is increasingly being recommended as a routine procedure in the investigation of any sudden unexpected death. ${ }^{25,26,68,69}$ Sudden death is often the sentinel event of $10 \%-40 \%$ of LQTS, as most genetic variant carriers are unaware that the disease is present. ${ }^{26,69-71}$ The importance of postmortem genetic testing lies not only in determining the cause of death at autopsy but also serves as a diagnostic tool in identifying relatives (of the deceased) at risk for the same inherited genetic disorder. ${ }^{26,29,69}$ Over $95 \%$ of cardiac genetic disorders (in the general population) are inherited as an autosomal-dominant trait. ${ }^{69}$ Furthermore, the risk for subsequent siblings dying from SUID is reported to be between 3.7-fold and 10-fold (although this is regarded as controversial by some). ${ }^{2}$

Various treatment modalities for channelopathies are available, with the three most common/effective being that of $\beta$-adrenergic blockers, antiarrhythmic agents and the use of implanted device therapy. ${ }^{13,28,63}$ Although $\beta$-adrenergic blockers are still considered the first line of therapy in LQTS, a lower efficacy in treatment for SCN5A variant-associated LQTS has been reported. ${ }^{13,28}$ Evidence obtained from both clinical and in vitro settings suggests a successful counteraction of mexiletine against the aberrant persistent $\mathrm{Na}^{+}$current, which ultimately shortens the QT interval in SCN5A pathogenic variation carriers. ${ }^{28,63}$ In addition, flecainide also proves to shorten QT intervals in many SCN5A pathogenic variation carriers; however, concerns regarding the safety of this specific therapy have been raised. ${ }^{13,28,63}$ Quinidine and sotalol, both class III-type antiarrhythmic agents, proved to be beneficial to patients diagnosed with BrS. ${ }^{13,28}$ Patients with LQTS and BrS seem to benefit significantly from implantable defribrillators, whereas patients suffering from conduction disorders were managed successfully with pacemaker implantation as treatment option. ${ }^{13,28,52,63}$

The profound value of existing treatment for these arrhythmic diseases may be best portrayed by Wilders' comparison of two similar case studies and their associated clinical outcomes. ${ }^{13}$ Both cases involved neonates with documented arrhythmias and a prolonged QT interval, though only one of the cases received treatment on presentation of clinical symptoms. ${ }^{13,72}$ The first case was reported by Southall et $a l^{72}$ on a neonate who presented with arrhythmias in utero and bradycardia for the first 9 days of life; however, on day 10, a normal heart rate was recorded and the baby was discharged from hospital. Unfortunately, the baby suffered a sudden and unexpected death 3 days later, which, after an autopsy investigation, remained unexplained. On retrospective analysis of the available ECG recordings, a substantial QT interval prolongation was observed. ${ }^{13,72}$ In contrast, a second neonate who also presented with arrhythmias in utero and a 24-hour ECG illustrating a prolonged QT interval with frequent premature ventricular beats received a $\beta$-blocker 
(propranolol), which proved to be successful in treatment. ${ }^{13,72}$ Since the disease is potentially treatable, the ability of molecular testing to identify these channelopathies as a cause of death in SUID cases will allow for testing and initiation of preventive therapies not exclusively to just family members at risk but even in future pregnancies. ${ }^{26,65,69}$ Unfortunately, as a consequence of the almost silent nature of the disorder (sudden death being the first 'symptom'), ${ }^{26,69-71}$ genetic testing would be difficult to implement as a preventative measure before any SUID occurrence or without strong suspicion due to known family history. The role of postmortem genetic testing in this age group will be to establish the prevalence of these variations in the general population.

\section{THE ROLE OF MOLECULAR TESTING}

Considering all the data, the question arises as to whether a routine postmortem genetic analysis should be implemented in all sudden infant deaths that remain unexplained after a thorough autopsy investigation.

First, as described by Skinner, ${ }^{52}$ the identification of pathogenic variations in SUID victims does not necessarily prove causality even if their clinical significance has been proven to be disease causing in other families or by in vitro testing. This leads to the old dictum where the forensic pathologists need to decide if the person died with the disease or as a result thereof. However, evidence exists (referenced throughout this paper) that SUID may be due, in a minority, to cardiac channelopathies such as long QT syndrome.

Second, the question arises as to what extent forensic pathologists are legally and ethically bound to conduct these tests. It can be argued that the forensic pathologists need to determine the cause, and in some cases the manner, of death. The next-of-kin in these cases might benefit tremendously from testing, which in some instances could include ECG screening followed by genetic testing. ${ }^{43,46,71}$ This would necessitate close working relationships between forensic pathologists and a team of other experts including molecular biologists, cardiologists and genetic counsellors. The importance of findings by forensic pathologists over the years has drastically led to the reduction of certain mortalities-for example, the implementation of restraint devices in road traffic accidents-and cannot thus be negated.

Third, in many instances, finances are not available to routinely conduct these tests. On average, screening only for variations in the SCN5A gene, which is reported to be found in $5.2 \%$ of SUID victims, ${ }^{13,28,52,63}$ would cost approximately US\$570 per case in South Africa (the cost of similar genetic testing may differ between countries). However, these costs will be dramatically reduced in the event of implementation of routine genetic testing in all unexplained SUID cases, as targeted genetic testing of known hotspot regions will be used instead of whole exome sequencing. Research should also focus on screening the general population to determine which variations occur naturally in any given population. A recent molecular study conducted on South African SUIDs (unpublished data) revealed eight specific exons of the SCN5A gene as definite hotspot regions particular to this population. In effect, the costs of postmortem genetic testing, refined to those eight hotspot regions, in a single SUID case, would amount to approximately US\$143. Considering the reduced costs, which should continue to decline due to advances in technology, one might argue that ethical issues far outweigh financial concerns with regard to targeted postmortem genetic testing in applicable SUID cases.
The question will always remain as to which genes should be tested for in each case. According to the Heart Rhythm Society/ European Heart Rhythm Association guidelines, targeted postmortem mutational analysis in all sudden unexpected deaths between 0 and 40 years of age is recommended. ${ }^{30,73}$ In countries such as Australia and New Zealand, all sudden and unexpected deaths are mandated to undergo targeted postmortem genetic testing. ${ }^{30,69}$ In 2015, the Swiss Society of Legal Medicine recommended that all sudden unexpected deaths under the age of 40 should be subjected to postmortem genetic testing. ${ }^{73}$ In a recent study conducted by Sanchez et al, ${ }^{73}$ next-generation sequencing (NGS) postmortem genetic analyses showed that in $13.4 \%$ of sudden unexplained death cases (between 0 and 10 years of age), a disease-causing variation linked to an inherited cardiac arrhythmic disorder (LQTS, BrS and CPVT) was identified and diagnosed as the cause of death. ${ }^{73}$ In the remaining $31.9 \%$ cases, in which variants considered possibly pathogenic could not be fully defined as the cause of death, a necessity for family members to consider further genetic evaluation was established. ${ }^{73}$ As a result of their findings, they recommend that NGS genetic analyses should be performed on all unexplained sudden deaths below the age of $40 .^{73}$

In our opinion, interdisciplinary centres should conduct large studies in order to attempt identifying the true incidence of these cases. Prospective and retrospective studies could be undertaken. At most large medicolegal death investigation centres (which are often linked to tertiary academic institutions), forensic pathologists have established archives of formalin-fixed, paraffin-embedded (FFPE) tissue samples, which can serve as a (sometimes only) source of material that contains critical genetic information valuable to molecular testing. ${ }^{74,75}$ Several studies have reported the successful, though not necessarily ideal, use of FFPE tissue samples in retrospective postmortem mutational analysis of previously admitted SUID cases. ${ }^{53,57,74,75}$ This raises an important issue pertaining to a possible difference in cost between the usage of FFPE tissue samples versus more traditional samples such as DNA extracted from blood. From experience working with FFPE tissue samples as a source of DNA for postmortem genetic testing, costs increase dramatically compared with using blood samples as the source of DNA. However, the rise in cost almost completely depended on factors associated with the incorrect conditions/circumstances surrounding the retention, fixation and storage of FFPE tissue samples. When prescribed guidelines were followed for the retention and fixation of FFPE tissue samples (fixed in formalin for a maximum of 24 hours, cleared in xylene and embedded in a paraffin block), DNA extraction and subsequent molecular applications were equal in quality, be it at lower concentrations, when compared with DNA extracted from blood. Thus, the difference in cost between using these two sources of genetic material for genetic testing may, in fact, be insignificant and therefore highlights the crucial importance of appropriate sampling/storage of all retained autopsy samples.

Combining resources and including all infants (regardless of the manner/cause of death) in testing for specific genetic variations could provide data on the most commonly encountered variations for each subset. Although this would most definitely be a very costly undertaking, identifying the specific genetic variations and their associated hotspot regions could prove cost-effective in the long term as more focused testing (which will be more affordable) could be undertaken.

Knowledge gained from the results of these tests could be imperative for adequate genetic counselling of parents of subsequent cases and provide closure to families who were previously informed that no cause of death was identified. This will assist in 
providing closure and planning options (such as genetic testing) for all siblings, adding significant value in the possible prevention of future similar cases to all individuals involved. ${ }^{43,75}$

Thus, ethical and reasonable justifications compel us to seek a molecular diagnosis of LQTS in an infant whose sudden death remains unexplained despite a thorough autopsy and ancillary investigations, and should therefore be considered in all medicolegal settings. ${ }^{52}$

Correction notice This paper has been amended since it was published Online First. Owing to a scripting error, some of the publisher names in the references were replaced with 'BMJ Publishing Group'. This only affected the full text version, not the PDF. We have since corrected these errors and the correct publishers have been inserted into the references.

Contributors All three authors were involved in the conception, writing and editing of this paper.

\section{Competing interests None declared.}

Provenance and peer review Commissioned; externally peer reviewed.

(c) Article author(s) (or their employer(s) unless otherwise stated in the text of the article) 2017. All rights reserved. No commercial use is permitted unless otherwise expressly granted.

\section{REFERENCES}

1 Moon RY, Horne RS, Hauck FR. Sudden infant death syndrome. Lancet 2007;370:1578-87.

2 Carroll R, Wood JN. Sudden unexpected infant death: a compassionate forensic approach to care. Clin Pediatr Emerg Med 2012;13:239-48.

3 Evans A, Bagnall RD, Duflou J, et al. Postmortem review and genetic analysis in sudden infant death syndrome: an 11-year review. Hum Pathol 2013;44:1730-6.

4 Centers for Disease Control and Prevention. Sudden unexpected infant death and sudden infant death syndrome. 2016 www.cdc.gov/sids/data.htm (accessed 8 Apr 2016).

5 Davis P, Price L, Heatman B, et al. Sudden Unexpected Death in Infancy-A Collaborative Thematic Review 2010-2012. London, UK: Public Health Wales NHS Trust, 2015.

6 Dettmeyer RB, Kandolf R. Cardiomyopathies—-misdiagnosed as Sudden Infant Death Syndrome (SIDS). Forensic Sci Int 2010;194:e21-e24.

7 Tester DJ, Ackerman MJ. Postmortem long QT syndrome genetic testing for sudden unexplained death in the young. J Am Coll Cardiol 2007;49:240-6.

8 Opdal SH, Rognum TO. Gene variants predisposing to SIDS: current knowledge. Forensic Sci Med Pathol 2011;7:26-36.

9 Kubuš $\mathrm{P}$, Janoušek J. Sudden cardiac death in children and young adultsepidemiology and prevention. Cor Vasa 2012;54:e223-e226.

10 Pasquale-Styles MA, Tackitt PL, Schmidt CJ. Infant death scene investigation and the assessment of potential risk factors for asphyxia: a review of 209 sudden unexpected infant deaths. J Forensic Sci 2007;52:924-9.

11 Dempers JJ, Coldrey J, Burger EH, et al. . The Institution of a Standardized Investigation Protocol for Sudden Infant Death in the Eastern Metropole, Cape Town, South Africa. J Forensic Sci 2016:61:1508-14.

12 Byard RW. Changing infant death rates: diagnostic shift, success story, or both? Forensic Sci Med Pathol 2013:9:1-2

13 Wilders R. Cardiac ion channelopathies and the sudden infant death syndrome. ISRN Cardiol 2012;2012:1-28.

14 Byard RW. SUDI or "undetermined": does it matter? Forensic Sci Med Pathol 2009;5:252-3.

15 Byard RW. Possible mechanisms responsible for the sudden infant death syndrome. J Paediatr Child Health 1991;27:147-57.

16 Moon RY. Task Force on Sudden Infant Death Syndrome. SIDS and other sleep-related infant deaths: expansion of recommendations for a safe infant sleeping environment. Pediatrics 2011;128:1030-9.

17 Kinney HC, Thach BT. The sudden infant death syndrome. N Engl J Med 2009:361:795-805.

18 Arnestad M, Crotti L, Rognum TO, et al. Prevalence of long-QT syndrome gene variants in sudden infant death syndrome. Circulation 2007;115:361-7.

19 Garcia AJ, Koschnitzky JE, Ramirez JM. The physiological determinants of sudden infant death syndrome. Respir Physiol Neurobiol 2013;189:288-300.

20 Glengarry JM, Crawford J, Morrow PL, et al. Long QT molecular autopsy in sudden infant death syndrome. Arch Dis Child 2014;99:635-40.

21 Filiano JJ, Kinney HC. A perspective on neuropathologic findings in victims of the sudden infant death syndrome: the triple-risk model. Biol Neonate 1994:65:194-7.

22 Guntheroth WG, Spiers PS. The triple risk hypotheses in sudden infant death syndrome. Pediatrics 2002;110:e64-6.
23 Paterson DS, Trachtenberg FL, Thompson EG, et al. Multiple serotonergic brainstem abnormalities in sudden infant death syndrome. JAMA 2006;296:2124-32.

24 Kinney HC, Richerson GB, Dymecki SM, et al. The brainstem and serotonin in the sudden infant death syndrome. Annu Rev Pathol 2009;4:517-50.

25 Wang D, Shah KR, Um SY, Yon Um S, et al. Cardiac channelopathy testing in 274 ethnically diverse sudden unexplained deaths. Forensic Sci Int 2014:237:90-9.

26 Stattin EL, Westin IM, Cederquist K, et al. Genetic screening in sudden cardiac death in the young can save future lives. Int J Legal Med 2016:130:59-66.

27 Randall BB, Paterson DS, Haas EA, et al. Potential asphyxia and brainstem abnormalities in sudden and unexpected death in infants. Pediatrics 2013;132:e161 6-e1625.

28 Crotti L, Celano G, Dagradi F, et al. Congenital long QT syndrome. Orphanet J Rare Dis 2008; $3: 18-16$

29 Davis AM, Glengarry J, Skinner JR. Sudden infant death: QT or not QT? That is no longer the question. Circ Arrhythm Electrophysiol 2016;9:e003859.

30 Tester DJ, Medeiros-Domingo A, Will ML, et al. Cardiac channel molecular autopsy: insights from 173 consecutive cases of autopsy-negative sudden unexplained death referred for postmortem genetic testing. Mayo Clin Proc 2012;87:524-39.

31 Schwartz PJ, Stramba-Badiale M, Segantini A, et al. Prolongation of the QT interval and the sudden infant death syndrome. $N$ Engl J Med Overseas Ed 1998:338:1709-14.

32 Zimmer T, Surber R. SCN5A channelopathies-an update on mutations and mechanisms. Prog Biophys Mol Biol 2008;98:120-36.

33 Michaud K, Fellmann F, Abriel H, et al. Molecular autopsy in sudden cardiac death and its implication for families: discussion of the practical, legal and ethical aspects of the multidisciplinary collaboration. Swiss Med Wkly 2009;139:712-8.

34 Keeton BR, Southall E, Rutter N, et al. Cardiac conduction disorders in six infants with "near-miss" sudden infant deaths. Br Med J 1977;2:600-1.

35 Ackerman MJ, Siu BL, Sturner WQ, et al. Postmortem molecular analysis of SCN5A defects in sudden infant death syndrome. JAMA 2001;286:2264-9.

36 Wedekind H, Bajanowski T, Friederich P, et al. Sudden infant death syndrome and long QT syndrome: an epidemiological and genetic study. Int I Legal Med 2006;120:129-37.

37 Plant LD, Bowers PN, Liu Q, et al. A common cardiac sodium channel variant associated with sudden infant death in African Americans, SCN5A S1103Y. J Clin Invest 2006;116:430-5.

38 Otagiri T, Kijima K, Osawa M, et al. Cardiac ion channel gene mutations in sudden infant death syndrome. Pediatr Res 2008;64:482-7.

39 Millat G, Kugener B, Chevalier P, et al. Contribution of long-QT syndrome genetic variants in sudden infant death syndrome. Pediatr Cardiol 2009;30:502-9.

40 National Society of Genetic Counselors. What is post mortem genetic testing? 2015 www.nsgc.org/postmortem (accessed 9 Dec 2016).

41 Kiehne N, Kauferstein S. Mutations in the SCN5A gene: evidence for a link between long QT syndrome and sudden death? Forensic Sci Int Genet 2007;1:170-4

42 Schwartz PJ, Ackerman MJ, George AL, et al. Impact of genetics on the clinical management of channelopathies. J Am Coll Cardiol 2013;62:169-80.

43 Ackerman MJ, Marcou CA, Tester DJ. Personalized medicine: genetic diagnosis for inherited cardiomyopathies/channelopathies. Rev Esp Cardiol 2013;66:298-307.

44 Monteforte N, Napolitano C, Priori SG. Genetics and arrhythmias: diagnostic and prognostic applications. Rev Esp Cardiol 2012;65:278-86.

45 Venetucci L, Garratt CJ. Cardiac channelopathies. Medicine 2014;42:591-4.

46 Vyas V, Lambiase PD. The investigation of sudden arrhythmic death syndrome (SADS) - the current approach to family screening and the future role of genomics and stem cell technology. Front Physiol 2013:4:1-12.

47 Nader A, Massumi A, Cheng J, et al. Inherited arrhythmic disorders: Iong QT and Brugada syndromes. Tex Heart Inst I 2007;34:67-75.

48 Hertz CL, Christiansen SL, Ferrero-Miliani L, et al. Next-generation sequencing of 100 candidate genes in young victims of suspected sudden cardiac death with structural abnormalities of the heart. Int I Legal Med 2016;130:91-102.

49 Human Gene Mutation Database. Genes—SCN5A [Internet]. 2013. Available at http://www.hgmd.cf.ac.uk/ac/gene.php?gene=SCN5A (accessed 2 Feb 2017).

50 Illikova V, Hlivak P, Hatala R. Cardiac channelopathies in pediatric patients-7-years single center experience. J Electrocardiol 2015;48:150-6.

51 Abriel H, Zaklyazminskaya EV. Cardiac channelopathies: genetic and molecular mechanisms. Gene 2013;517:1-11.

52 Skinner JR. Is there a relation between SIDS and long QT syndrome? Arch Dis Child 2005;90:445-9.

53 Ackerman MJ. State of postmortem genetic testing known as the cardiac channel molecular autopsy in the forensic evaluation of unexplained sudden cardiac death in the young. Pacing Clin Electrophysiol 2009;32 Suppl 2:S86-S89.

54 Wang Q, Li Z, Shen J, et al. Genomic organization of the human SCN5A gene encoding the cardiac sodium channel. Genomics 1996;34:9-16.

55 de la Grandmaison GL. Is there progress in the autopsy diagnosis of sudden unexpected death in adults? Forensic Sci Int 2006;156:138-44.

56 Schwartz PJ, Priori SG, Dumaine $R$, et al. A molecular link between the sudden infant death syndrome and the long-QT syndrome. N Engl J Med 2000;343:262-7. 
57 Basso C, Calabrese F, Corrado D, et al. Postmortem diagnosis in sudden cardiac death victims: macroscopic, microscopic and molecular findings. Cardiovasc Res 2001;50:290-300.

58 Campuzano 0, Sanchez-Molero 0, Allegue C, et al. Post-mortem genetic analysis in juvenile cases of sudden cardiac death. Forensic Sci Int 2014;245:30-7.

59 Schwartz PJ, Stramba-Badiale M, Segantini A, et al. Prolongation of the QT interval and the sudden infant death syndrome. N Engl J Med 1998;338:1709-14.

60 Schwartz PJ, Priori SG, Bloise R, et al. Molecular diagnosis in a child with sudden infant death syndrome. Lancet 2001;358:1342-3.

61 Weese-Mayer DE, Ackerman MJ, Marazita ML, et al. Sudden Infant Death Syndrome: review of implicated genetic factors. Am J Med Genet A 2007;143A:771-88.

62 Madea B. Sudden death, especially in infancy-improvement of diagnoses by biochemistry, immunohistochemistry and molecular pathology. Leg Med 2009;11 Suppl 1:36-42.

63 George AL. Inherited disorders of voltage-gated sodium channels. J Clin Invest 2005; 115:1990-9.

64 Chugh SS, Reinier K, Teodorescu C, et al. Epidemiology of sudden cardiac death: clinical and research implications. Prog Cardiovasc Dis 2008;51:213-28.

65 Doolan A, Langlois N, Chiu C, et al. Postmortem molecular analysis of KCNQ1 and SCN5A genes in sudden unexplained death in young Australians. Int J Cardiol 2008;127:138-41.

66 Millat G, Chevalier P, Restier-Miron L, et al. Spectrum of pathogenic mutations and associated polymorphisms in a cohort of 44 unrelated patients with long QT syndrome. Clin Genet 2006;70:214-27.
67 GenAtlas. Genes-SCN5A. 2013 www.genatlas.medicine.univ-paris5.fr/fiche.php? symbol=SCN5A (accessed 21 Nov 2015).

68 Platt MW, Blair PS, Fleming PJ, et al. A clinical comparison of SIDS and explained sudden infant deaths: how healthy and how normal?. Arch Dis Child 2000;82:98-106

69 Semsarian C, Ingles J, Wilde AAM. Sudden cardiac death in the young: the molecular autopsy and a practical approach to surviving relatives. Eur Heart J 2015;36:1290-6.

70 Deo R, Albert CM. Epidemiology and genetics of sudden cardiac death. Circulation 2012;125:620-37.

71 Schwartz PJ, Crotti L. Can a message from the dead save lives? J Am Coll Cardiol 2007:49:247-9.

72 Southall DP, Arrowsmith WA, Stebbens V, et al. QT interval measurements before sudden infant death syndrome. Arch Dis Child 1986;61:327-33.

73 Sanchez 0, Campuzano 0, Fernández-Falgueras A, et al. Natural and undetermined sudden death: value of post-mortem genetic investigation. PLoS One 2016;11:e0167358.

74 Gilbert MT, Haselkorn T, Bunce M, et al. The isolation of nucleic acids from fixed, paraffin-embedded tissues-which methods are useful when? PLoS One 2007; 2:e537-12

75 Carturan E, Tester DJ, Brost BC, et al. Postmortem genetic testing for conventional autopsy-negative sudden unexplained death: an evaluation of different DNA extraction protocols and the feasibility of mutational analysis from archival paraffinembedded heart tissue. Am J Clin Pathol 2008:129:391-7. 